International Journal of Engineering \& Technology, $7(2.28)(2017) 119-122$
International Journal of Engineering \& Technology
SPC
Website: www.sciencepubco.com/index.php/IJET
Research paper

\title{
Virtual reality training system based on lower limb rehabilitation robot
}

\author{
Hongbo Wang ${ }^{1}$, Musong Lin ${ }^{1}$, Zhennan Jin ${ }^{1}$, Xincheng Wang ${ }^{1}$, \\ Jianye Niu' ${ }^{1}$, Hongfei Yu ${ }^{1}$, Lili Zhang ${ }^{1}$, Luige Vladareanu ${ }^{2 *}$ \\ ${ }^{1}$ Parallel Robot and Mechatronic System Laboratory of Hebei Province and \\ Key Laboratory of Advanced Forging \& Stamping Technology and Science of Ministry of \\ Education, Yanshan University, Qinhuangdao, 066004, China \\ ${ }^{2}$ Romanian Academy, Institute of Solid Mechanics, Bucharest, Romania \\ *Corresponding authorE-mail: luige.vladareanu@vipro.edu.ro; luigiv2007@yahoo.com.sg
}

\begin{abstract}
This paper presents a virtual reality training system for the lower limb rehabilitation robot, which can simulate the bike riding and encourage patients to join in the recovery training through the built-in competitive game. The virtual reality training is a variable speed active training under the constraint trajectory, and it has adapting training posture function which can provide individual riding training track according to the legs length of patients. The movement synchronization between the robot and virtual model is achieved by interaction control strategy, and robot can change the training velocity based on the signal from feedback terrains in game. A serious game about bike match in forest was designed, and the user can select the training level as well as change perspective through the user interface. The training can be paused at any time, and the timer function could reflect the recovery of patient.
\end{abstract}

Keywords: rehabilitation robot; virtual reality; interact strategy; serious game.

\section{Introduction}

As aging society comes to many counties in the world, the health of elderly has become a focus problem [1-2]. Stroke is a common disease in the elderly which has a high morbidity and disability [3], and the rehabilitation training based on neural plasticity is regarded as an effective method to stroke sequela [4-6]. Traditional rehabilitation need a long term one-on-one treatment which costs too much human resources, and it cannot maintain a stable intensive. Since the training process is too boring and simple, it is hard to attract patients and receive active cooperation. However, the combination of robotics and VR (Virtual Reality) could properly solve these problems.

Serious game is a kind of application designed for a primary purpose other than pure entertainment, and it is generally referred to video games based on VR technology which are commonly used in defense, education, scientific exploration, health care. As the positive effects of VR games in rehabilitation are shown by various scientific studies [7-9], the VR applications in rehabilitation robots have been the focus of researchers in various countries. MIT-Manus is the first widely known limb rehabilitation robot with simple VR system, and a robot named GENTLE/s with virtual interaction scenes was designed by University of Reading [1011]. A 6-DOF (Degree Of Freedom) rehabilitation robot with VR function was developed by Osaka University [12]. A VR-based rehabilitation robot was developed by Tianjin University of Technology, which could make the training process visual and interactive [13].
This paper present a VR training system based on a lower limb rehabilitation robot, and it is shown as follow: Part II is the introduction of the rehabilitation robot and the sensors. In part III, the design of VR training system is presented. Part IV is the build process of VR game scenes and game functions. The experiment data of VR training is shown in the last part V.

\section{Application device}

\subsection{Lower limb rehabilitation robot}

The appearance of LLRR (Lower Limb Rehabilitation Robot) is shown in Fig. 1. LLRR was designed as a modular structure, and it consist of the left machine leg, the right machine leg, the separable chair and the electric box.

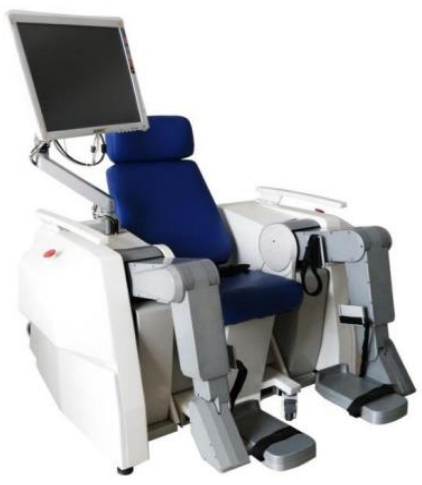

Fig. 1: Lower limb rehabilitation robot 
Each machine leg owns 3-DOF and contains hip joint, knee joint and ankle joint. The machine leg could be divided into the thigh part and the shank part, and the length of each part could be changed electronically to meet the various legs length of patients from $1.5 \mathrm{~m}$ to $1.9 \mathrm{~m}$. To satisfy the different shapes of patients, the width between two legs could be adjusted automatically. A separable chair used for sitting/lying training and patients transfer was designed. LLRR could realize active training, passive training, teaching training, VR (Virtual Reality) training and others.

\subsection{Necessary sensor element}

The torque and pressure sensors equipped on LLRR are shown in Fig. 2. Four torque sensors are installed in hip and knee joints which could receive torque data constantly from the joints. The joint torque data is the necessary judgment of the active training and the VR training. Foot pressure data, which is collected by sensors equipped in the foot pedal, could transform into the acceleration factor used for controlling training velocity of machine legs terminal.

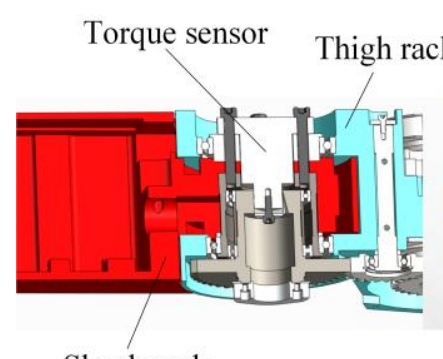

Shank rack

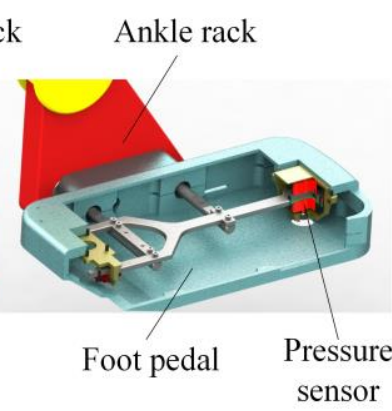

Fig. 2: Necessary sensor element

\section{Virtual reality training system}

To cooperate with the VR software and simulate the riding body feeling, a VR training system was designed. VR training is an improved kind of active training, it includes robot control, adapting training posture and interaction control strategy. The robot control is similar to the normal active training, which has been studied in paper [14]

\subsection{Design of adapting training posture function}

Based on the research of bike mechanism and riding body posture, as shown in Fig. 3, adapting posture function was built.

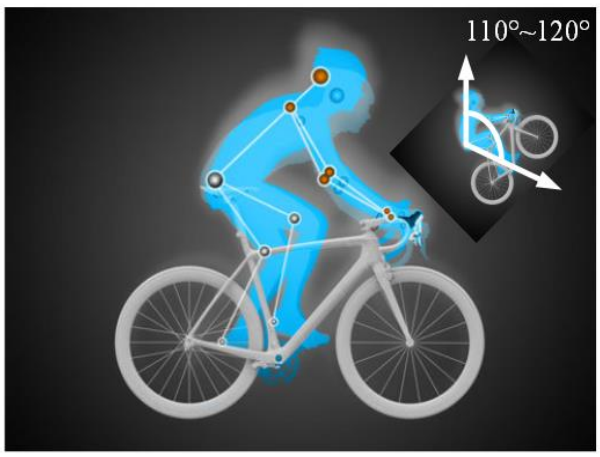

Fig. 3: Riding body posture

The function can provide individual terminal trajectory according to the different legs length of patients. Based on alternative tracks in workspace, it could select suitable track for patients which could make training closer to real bike riding (Fig. 4).
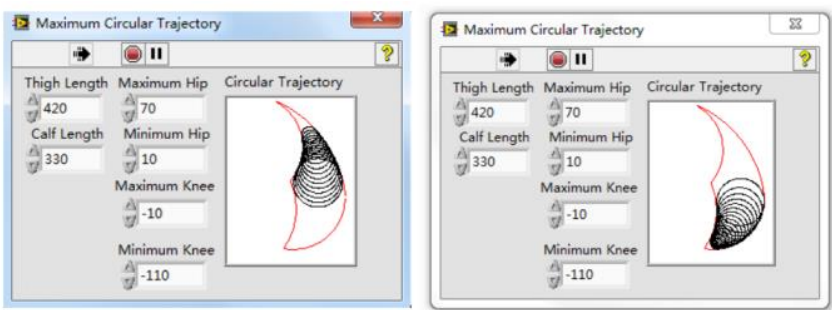

Fig. 4: Calculated circular trajectory

\subsection{Interaction control strategy}

Interaction control strategy is a necessary link between the robot and the VR software, and it mainly contains the model synchronization and feedback terrains. The strategy block diagram of interaction control is shown in Fig. 5.

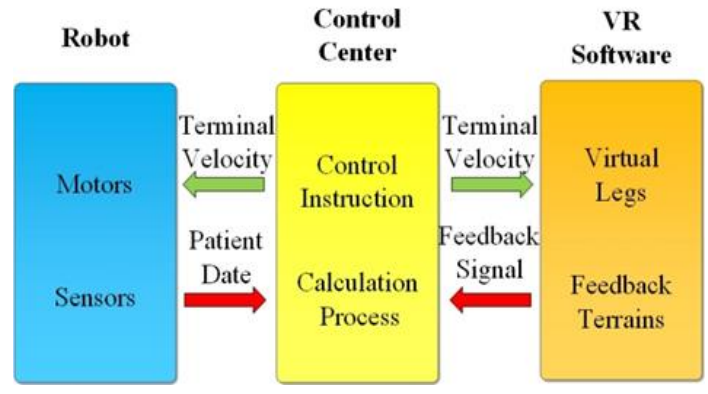

Fig. 5: Interaction control strategy

Comparing the sensors data with calculated data, the patient movement intention data could be determined. The torque intention is defined as the judge factor, and the robot will begin to run with default terminal velocity when judge factor exceeds the preset threshold. The pressure intention works as an acceleration factor when it exceeds the threshold, and there is a linear relationship between the factor and the value added on default velocity.

Final terminal velocity is used for LLRR control, and it is sent to VR software for model action synchronization. Meanwhile, there are some different feedback terrains set up in the VR riding game, such as hill and obstacle road. When the virtual character model in those terrains upon, the robot will receive the feedback signal from the VR software and then change the machine legs running speed depending on different conditions.

\section{Virtual reality software design}

\subsection{Virtual scene bulid}

Based on the game development engine Unity3D, the VR riding game was built. The planning of the virtual scene not only meets the requirements of exercise intensity for rehabilitation training, but also could stimulate the nervous system of the patients and has a good influence on psychological of patients. This scene properly meets the outdoors walking desire of the patient with walking problems.

An outdoor riding match scene with green background tone and plenty sunlight is shown in Fig. 6, and it could provide a relaxing virtual environment for patients.

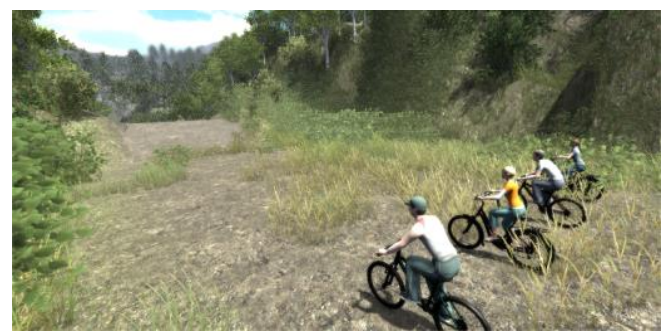

Fig. 6: Match scene in game 
Riding game has 4 character models, except one controlled by the patient, 3 models are NPC (Non-Player Character) with different actions controlled by the computer. Multi-characters could avoid loneliness, while match training has properly entertainment and competitiveness.

The whole road is about 600 meters, a single match will take 2 or 3 minutes. There are 2 obstacle areas and a hill on the road, and the stimulus strength of training could be changed when the model goes through these areas.

\subsection{Game function design}

VR game software cannot run without scripts, and each component or model in the game need a relative script at least. The main scripts are shown as follows.

1) User Interface: Used for game start, level select, software close and other buttons.

2) Model synchronization: Control the virtual legs move same as the real legs based on robot terminal velocity.

3) Model movement: According to terminal velocity, calculate speed and control model move forward along straight road.

4) NPC action: Control models run in preset parameters when game start, and NPC could run in 4 levels speed based on the difficulty choice in the title screen.

5) Feedback trigger: Constantly monitor positions of 4 models, if a model enter the feedback areas it will send signal to robot until the model leave areas.

6) Signal I/O: Build temp files used for writing and loading signal by robot and software.

7) Pause and timer: Game and robot could be paused at any time when training start, it could also record the time since the game start.

8) Camera: Game screen could be switched between the first-person perspective and the third-person perspective when the game start (Fig.7).

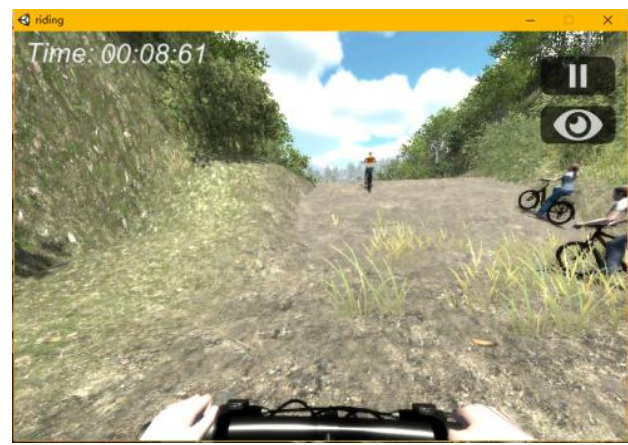

Fig. 7: First-person perspective

The connections between scripts and models were built, and scripts working condition in the simple scene was tested as shown in Fig. 8. After debugging, all components were imported into the completed scene.

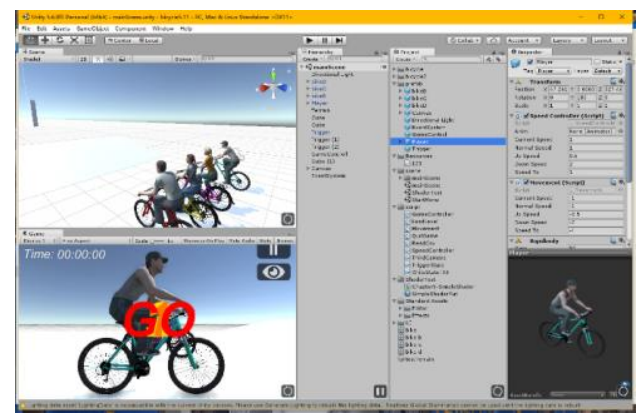

Fig. 8: Function test

\section{Virtual reality training experiment}

\subsection{Model synchronization test}

The movements of machine legs and virtual legs were recorded through the video. Due to the deflection between the training posture and the real riding posture, the movements are not exactly same as shown in Fig. 9. But the time costs of both legs reaching the lowest point in their own circle track are same, the synchronization between the robot and the model is properly achieved.

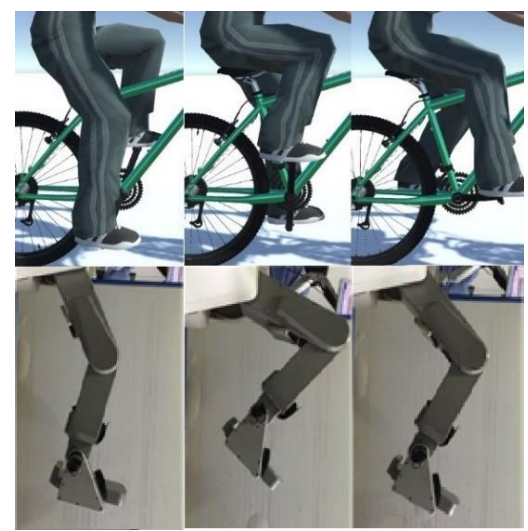

Fig. 9: Screenshot of synchronization test

\subsection{Feedback terrains test}

The sensors data is set as a fixed value, and the robot terminal velocity is recorded when the model goes through the feedback terrains in the game. The recording data was transformed into a graph shown in Fig. 10. The result shows that the patient need to pay more efforts or reduce the training speed when the character model goes into the difficult feedback areas, and the opposite effect will occur in easy areas.

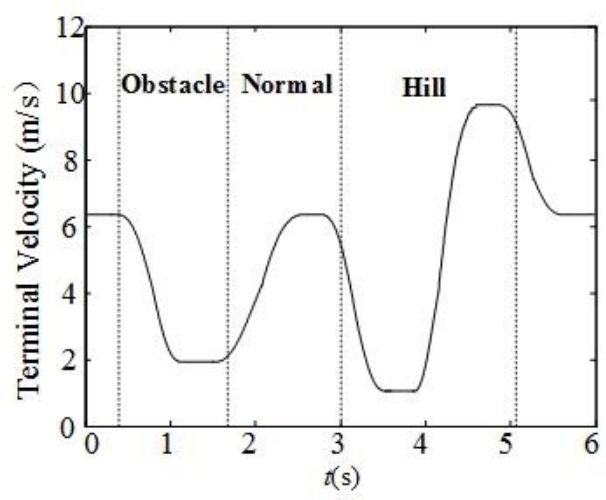

Fig. 10: Feedback terrains test

\section{Conclusion}

Based on the lower limb rehabilitation robot, a virtual reality training system with competitive game was designed, which could simulate the bike riding and encourage patients to join in the recovery training. It can realize movement synchronization between the robot legs and the virtual model, and the robot can vary terminal velocity according to the signal of feedback terrains in the game. The system can select the suitable training trajectory based on the legs length of patients before training, and the training can be paused by patients or doctors at any time while training. Doctors could switch VR training difficulty according to the recovery of patients, and the recovery could be reflected through the timer function. 


\section{Acknowledgement}

This work was developed with the support of "Joint Laboratory of Intelligent Rehabilitation Robot" collaborative research agreement between Yanshan University, China and Romanian Academy by IMSAR, RO by China Science and Technical Assistance Project for Developing Countries (KY201501009).

\section{References}

[1] Feng Y.F., Wang H.B., Lu T.T., et al (2016), Teaching training method of a lower limb rehabilitation robot. Int. J. Adv. Robot Syst., Vol. 13, No. 2, pp. 1-11.

[2] Ozkul F., Barkana D.E. (2013), Upper-extremity rehabilitation robot RehabRoby: methodology, design, usability and validation. Int. J. Adv. Robot Syst., Vol. 10, No. 1, pp. 1-13.

[3] Wei W.Q., Hou Z.G., Cheng L., et al (2016), Toward patients' motion intention recognition: dynamics modeling and identification of iLeg - an LLRR under Motion constraints. IEEE Transactions on Systems Man \& Cybernetics Systems, Vol. 46, No. 7, pp. 980-992.

[4] Fouad K., Krajacic A., Tetzlaff W (2011), Spinal cord injury and plasticity: opportunities and challenges. Brain Research Bulletin, Vol. 84, No. 4, pp. 337-342.

[5] Behrman A.L., Harkema S.J. (2000), Locomotor training after human spinal cord injury: a series of case studies. Physical therapy, Vol. 80, No. 7, pp. 688-700.

[6] Fouad K., Tetzlaff W. (2012), Rehabilitative training and plasticity following spinal cord injury. Experimental Neurology, Vol. 235, No. 1, pp. 91-99.

[7] Saini S., Rambli D.R.A., Sulaiman S., et al (2012), A low-cost game framework for a home-based stroke rehabilitation system. International Conference on Computer \& Information Science, Vol. 1, pp. 55-60.

[8] Holden M.K. (2005), Virtual environments for motor rehabilitation: review. Cyberpsycholo. Behav., Vol. 8, No. 3, pp. 187-211.

[9] Schönauer C., Pintaric T., Kaufmann H. (2011), Full body interaction for serious games in motor rehabilitation. Augm. Human Int. Conf., Vol. 2, pp. 1-8.

[10] Krebs H., Celestino J., Williams D., et al (2004), 24 A Wrist Extension for MITMANUS. Advances in Rehabilitation Robotics, Vol. 306, pp. 377-390.

[11] Loureiro R., Amirabdollahian F., Topping M., et al (2003), Upper Limb Robot Mediated Stroke Therapy - GENTLE/s Approach. Reading: Autonomous Robots, Vol. 15, No. 1, pp. 35-51.

[12] Furusho J., Li C.Q., Yamaguchi Y. (2005), A 6-DOF rehabilitation machine for upper limbs including wrists using ER actuators. Me chatronics and Automation, 2005 IEEE International Conference IEEE, Vol. 2, pp. 1033-1038.

[13] Wei W., Guo S., Zhang W., et al (2013), A novel VR-based upper limb rehabilitation robot system. ICME International Conference on Complex Medical Engineering IEEE, pp. 302-306.

[14] Feng Y.F., Wang H.B., Lu, T.T., et al (2016), Teaching training method of a lower limb rehabilitation robot. International Journal of Advanced Robotic Systems, Vol. 13, No. 2, pp. 1-11. 